\title{
La universidad como espacio para la prevención de la violencia de género. Una propuesta didáctica a través de la literatura española (siglos XIX-XXI)'
}

\author{
The University as a Space to Prevent Gender - Based Violence - \\ A Teaching Approach through Literature from Spain (19th to 2 I st Centuries)
}

\author{
CLARA EUGENIA PERAGÓN LÓPEZ \\ Universidad de Córdoba \\ España \\ cperagon@uco.es
}

(Recibido: I2-O4-20I9; aceptado: $3 \mathrm{O}-\mathrm{O} 3-2 \mathrm{O} 2 \mathrm{O}$ )

Resumen. A partir del reconocido potencial de la literatura como producto histórico, social y cultural, este artículo se centra en la lectura y explotación didáctica de una selección de cuentos de Emilia Pardo Bazán y de las novelas Algún amor que no mate (Dulce Chacón) y La segunda mujer (Luisa Castro) que, desde el siglo XIX hasta la actualidad, abordan la temática de la violencia contra la mujer. La primera parte constituye una revisión del correspondiente marco legal, así como de algunas investigaciones de ámbito nacional que han ido surgiendo desde la aprobación de la Ley Orgánica I/2004, de 29 de diciembre, de Medidas de Protección Integral contra la Violencia de Género, donde se destaca la necesidad de promover, desde la universidad, la formación en este ámbito. En la segunda parte se desarrolla una propuesta didáctica transversal orientada al alumnado universitario y estructurada según los elementos curriculares y metodológicos propios de toda planificación. Con este proyecto se pretende ofrecer una herramienta para la formación y sensibilización en torno a esta lacra social, a la vez que se revaloriza el papel de los textos literarios, cuya lectura permitirá a sus receptores la construcción crítica, fundamentada y compartida de su propio conocimiento al respecto.

Palabras clave: violencia; mujer; universidad; literatura; España.
Abstract.Blased on the recognized potential of Literature as a source for the study of historical, cultural and social topics, this article posits a reading and a teaching use of some works of Literature from Spain that, since the 19th century to present, address the issue of violence against women: a selection of stories by Emilia Pardo Bazán and two novels by the writers Dulce Chacón (Algún amor que no mate) and Luisa Castro (La segunda mujer). The first part of this project focuses on the revision of the legal framework in relation to the subject, as well as on some of the national investigations that have been appearing since the approval of the Organic Law I/2004, of December 29, on Comprehensive Protection Measures against Gender-based Violence, that highlights the need to promote, from the university, the training of students on gender equality and non-discrimination. The second part presents a teaching approach aimed at university students that pursue, on a transversal basis, the prevention and awareness of this matter, according to the curricular and methodological elements of lesson planning. This project aims to revalue the role of literary texts as a tool for training and raising awareness about this social scourge.

Keywords: violence; woman; university; literature; Spain.

\footnotetext{
${ }^{\text {I }}$ Para citar este artículo: Peragón López, Clara Eugenia (2020). La universidad como espacio para la prevención de la violencia de género. Una propuesta didáctica a través de la literatura española (siglos XIX-XXI). Alabe 22 . [www.revistaalabe.com] DOI: IO.I5645/Alabe2O20.22.6
} 


\section{Introducción}

La Cuarta Conferencia Mundial sobre la Mujer, celebrada en Beijing en I995, precedida de las acontecidas en Ciudad de México (I975), Copenhague (I980) y Nairobi (I985), constituyó un decisivo punto de inflexión en relación con el tema de la igualdad de género y la defensa de los derechos de las mujeres y de las niñas a nivel mundial. La Declaración y Plataforma de Acción de Beijing (I995), texto de referencia aprobado por I89 estados miembros, supuso la concreción de una serie de compromisos en torno a los temas tratados, con una clara voluntad política de visibilizarlos e introducir cambios concretos en cada uno de los países participantes en los que, veinticuatro años después, se sigue trabajando, dada la persistencia de significativos niveles de desigualdad entre hombres y mujeres.

La Plataforma de Acción, en su capítulo III, abarca doce esferas, denominadas "de especial preocupación", para las que se establecen objetivos estratégicos y medidas concretas. Entre ellas, destacamos la correspondiente a "La violencia contra la mujer"; una expresión de la que se ofrece una clarificadora definición en los siguientes términos:

[...] todo acto de violencia basado en el género que tiene como resultado posible o real un daño físico, sexual o psicológico, incluidas las amenazas, la coerción o la privación arbitraria de la libertad, ya sea que ocurra en la vida pública o en la privada (Naciones Unidas, 1995: 86).

Del mismo modo, se alude a las distintas formas en las que se manifiesta la violencia de género y a la histórica superioridad del hombre sobre la mujer, así como a la dominación y discriminación de esta última como claros antecedentes de una lacra social que, en pleno siglo XXI, se mantiene en una alarmante vigencia, lo que deriva, como también se declara, en la privación de los derechos y libertades fundamentales de la mujer.

Si nos centramos en las "Medidas que han de adoptarse", en relación con el primer objetivo que se plantea (D.r. "Adoptar medidas integradas para prevenir y eliminar la violencia contra la mujer"), se advierte sobre la necesidad de condenar este tipo de violencia, no cometer actos de esta índole, introducir sanciones penales, civiles, laborales y administrativas en las leyes nacionales, formular y aplicar planes de acción para erradicar la violencia, etc. En cuanto al ámbito de la educación, se determina la adopción de pautas para "modificar los modelos de conducta sociales y culturales de la mujer y el hombre, y eliminar los prejuicios y las prácticas consuetudinarias y de otro tipo basadas en la idea de la inferioridad o la superioridad de uno de los sexos [...]" (Naciones Unidas, I995: 92). En la misma línea, también se subraya la necesidad de promover y financiar campañas de sensibilización ante la que se considera una violación de los derechos humanos.

En las medidas relativas al objetivo D.2. ("Estudiar las causas y las consecuencias de la violencia contra la mujer y la eficacia de las medidas de prevención”) se insiste en la importancia de iniciar y apoyar estudios e investigaciones relacionados con el tema y 
difundir ampliamente los resultados obtenidos. De la misma manera, se hace referencia al papel de los medios de comunicación, a los que se alienta "a que examinen las consecuencias de los estereotipos basados en el género [...] y a que adopten medidas para eliminar esas imágenes negativas con miras a promover una sociedad sin violencia” (Naciones Unidas, r995: 97).

Sin duda, estas y otras recomendaciones marcaron una hoja de ruta para establecer una legislación en la que se protegieran los derechos de las mujeres ${ }^{2}$. Y fue precisamente en España donde, de manera pionera en Europa y de forma unánime, se aprobó la denominada Ley Orgánica I/2004, de 29 de diciembre, de Medidas de Protección Integral contra la Violencia de Género. Con un carácter integrador y multidisciplinar, la Ley se centra, entre otros ámbitos, en el educativo. De esta forma, el artículo 4 está dedicado a los "Principios y valores del sistema educativo". Concretamente, el apartado 7 estipula que las universidades "incluirán y fomentarán en todos los ámbitos académicos la formación, docencia e investigación en igualdad de género y no discriminación de forma transversal". Asimismo, el artículo 7 establece la necesidad de que las administraciones educativas adopten medidas para que, en los planes de formación inicial y permanente del profesorado, se contemple una formación específica en materia de igualdad con el fin de poder abordar las acciones correspondientes ${ }^{3}$.

Ya en el ámbito autonómico, tres años más tarde entra en vigor en Andalucía la Ley I3/2007, de 26 de noviembre, de medidas de prevención y protección integral contra la violencia de género. En el artículo ı6, sobre “Enseñanza Universitaria”, se especifican los aspectos que constituyen el marco legal sobre el que se asienta la propuesta didáctica que presentaremos con posterioridad. En este sentido, se insta al fomento de contenidos que permitan promover las relaciones de igualdad entre hombres y mujeres y la prevención de la violencia de género en los estudios universitarios de grado y en los programas de posgrado orientados, sobre todo, a la formación de profesionales de la enseñanza y de los medios de comunicación.

Desde la aparición de la Ley Orgánica de 2004, el creciente interés suscitado por el tema de la violencia de género ha repercutido en el desarrollo de investigaciones, muy escasas hasta ese momento, en las que se analiza, desde áreas de conocimiento y perspectivas diversas, su posible presencia en las universidades españolas, las distintas formas en las que se manifiesta y las creencias, percepciones y actitudes de los miembros de la comunidad universitaria ante esta realidad. A continuación, realizaremos un recorrido general por algunos estudios que han ido surgiendo desde el mencionado año hasta la actualidad, con el fin de conocer sus planteamientos y los aspectos concretos que se abordan en cada caso.

\footnotetext{
${ }^{2}$ Para un análisis pormenorizado de este documento y de los avances conseguidos tras la Conferencia, véase Giménez (2007).

3 Recientemente, Díez; Cerezo y Benítez (20I8) han analizado la efectividad y eficiencia de las iniciativas que se proponen en la Ley.
} 
En este sentido, comenzaremos centrándonos en la primera investigación que analiza la incidencia de la violencia de género en el contexto universitario español. Se trata del proyecto Violencia de género en las universidades españolas, financiado por el Ministerio de Igualdad-Instituto de la Mujer (Plan Nacional I+D+I 2004-2007), en el que participaron investigadores de ocho universidades. Ante la detectada escasez de medidas de atención y prevención sobre la problemática de la violencia de género en España, este proyecto acomete, además de un trabajo empírico analítico realizado sobre seis universidades españolas, una revisión de las investigaciones llevadas a cabo en centros internacionales de reconocido prestigio, así como de las medidas implementadas en los mismos para superar esta lacra social. Con todo ello, el objetivo fundamental que se persigue es, por un lado, plantear un estado de la cuestión sobre la literatura científica publicada sobre el tema a nivel internacional y, por otro, recoger ejemplos que constituyan modelos de buenas prácticas para desarrollar planes de actuación inexistentes en los años en los que se desarrolla el proyecto ${ }^{4}$.

En esta misma línea, destacan una serie de aportaciones de marcado carácter teórico (Oliver y Valls, 2004, Burgués; Oliver; Redondo y Serrano, 2006) que van dejando paso a otras donde se analiza la prevalencia de comportamientos violentos de carácter físico o psicológico de los estudiantes durante las relaciones de noviazgo en universidades de la Comunidad de Madrid (Muñoz; Graña; O`Leary y Lozano, 20O7), en la Universidad de Huelva (Hernando; García y Montilla, 2OI2), en la de Oviedo (García; Fernández; Rodríguez; López y Lana, 2OI3) y en la de Málaga (Fernández, 2OI4). Otros trabajos se centran en las universidades gallegas (Vázquez; Torres; Otero; Blanco y López, 2OIO), en la Universidad de Lérida (Calero; Molina; Bardina; Murillo y Rodríguez, 20I3), en la de Valencia (González y Mora, 20I4) y en la de Burgos (Tapia, 2OI5). Ya en 2OI6, Llorent y Sianes presentan los resultados de un sondeo para conocer la percepción del alumnado del Grado de Pedagogía de la Universidad de Sevilla sobre los denominados "micromachismos coercitivos" en su entorno. Tales formas de violencia se dan de manera recurrente en las relaciones de pareja y están estrechamente relacionadas con la violencia psicológica, lo que justifica la necesidad de identificarlas y visibilizarlas.

En líneas generales, los estudios referidos confirman que, efectivamente, la universidad española está afectada por la violencia de género, que determinadas situaciones no son reconocidas como tales y que existe una reticencia a la denuncia, siendo la inseguridad, el miedo o la educación recibida algunos de los factores condicionantes. Todo ello no hace sino corroborar la necesidad de una intervención educativa, demandada también explícitamente por el alumnado, muy especialmente cuando se trata de titulaciones que forman a futuros docentes, dando así respuesta a los requerimientos legales anteriormente expuestos. Según se desprende de las conclusiones del proyecto Incidencia de la

\footnotetext{
${ }^{4}$ Algunos de los resultados se han ido publicando en diversos artículos. Destacamos los siguientes: Valls; Oliver; Sánchez; Ruiz y Melgar 2007, Aguilar; Alonso; Melgar y Molina, 2OO9, Larena y Molina, 2OIO. Años más tarde, en 2OI3, surge otro estudio de ámbito estatal titulado La juventud universitaria ante la igualdad y la violencia de género. Se trata de una investigación liderada por Díaz-Aguado y realizada en colaboración entre la Unidad de Psicología Preventiva de la Universidad Complutense de Madrid y la Delegación de Gobierno para la Violencia de Género del Ministerio de Sanidad, Servicios Sociales e Igualdad. En este proyecto participaron doce universidades españolas.
} 
Ley Integral contra la Violencia de Género en la Formación Inicial del Profesorado ${ }^{5}$, en las facultades de Educación no se está llevando a cabo una formación obligatoria específica en este ámbito. Resulta pues, fundamental, seguir trabajando y visibilizando estos contenidos para lo que, según se apunta, las buenas prácticas de universidades de otros países constituyen un modelo que se podría tomar como referencia (Santos; Bas e Iranzo, 20I2).

No obstante, transcurrido algún tiempo desde el estudio anterior, y a falta de actualizar la información, habría que puntualizar que el panorama es otro muy distinto cuando se trata de la formación permanente en el ámbito universitario. Ya en aquellos años, y de manera creciente en la actualidad, son numerosos los congresos, jornadas, seminarios, simposios, etc. centrados en la violencia de género. Asimismo, habría que reivindicar la importante labor llevada a cabo por las unidades de igualdad de las universidades españolas, sin olvidar el progresivo aumento del número de estudiantes que deciden indagar en este tema a través de sus TFG, TFM o tesis doctorales.

Ante este panorama, queda probada la atención que esta cuestión suscita. Sin embargo, resultan muy escasas las aportaciones basadas en propuestas didácticas que orienten al profesorado en el procedimiento que permita la consecución de los principios postulados por las leyes correspondientes (Moreno, 2OIO, Romero, 2OI7). Por todo ello, con la propuesta didáctica que presentamos en este artículo pretendemos contribuir, de algún modo, a la ampliación de un horizonte en el que, de manera transversal y a través de algunas obras de la Literatura Española, las aulas universitarias se conviertan en espacios de prevención, sensibilización y formación en torno a temas sociales.

\section{La Literatura y la violencia de género}

La Literatura, y más concretamente el género narrativo, fiel reflejo, en muchos casos, de la historia, la sociedad y la cultura de distintas épocas, se ha ido configurando como una fuente indispensable para conocer determinados ámbitos de la realidad no consignados en otro tipo de documentos. Cuando el autor se enfrenta a la página en blanco, no lo hace desde la abstracción ni desde su propia individualidad, sino claramente influenciado por una realidad social que condicionará su posición, más o menos objetiva, ante los hechos que se dispone a relatar ${ }^{6}$.

La violencia de género, como tema social, la encontramos plasmada, aunque no con demasiada profusión, en obras literarias de diferentes épocas, la mayoría de ellas escritas por mujeres?. De este modo, y al hilo de lo argumentado con anterioridad,

\footnotetext{
5 Proyecto coordinado por el Centro de Investigación en Teorías y Prácticas Superadoras de Desigualdades (CREA) y la Universidad de Barcelona (Ministerio de Igualdad-Instituto de la Mujer. Plan Nacional I+D+[ 2OO7-2OIO).

6 Textos clásicos sobre este tema serían las obras de Lukács (I973), Goldmann (I975) y Bajtín (I99I).

7 Uno de los primeros episodios documentados pertenece al Cantar de Mío Cid. En él se recrea, en la conocida escena de "La afrenta de Corpes" (cantar tercero), el cruel y despiadado maltrato físico sufrido por doña Elvira y doña Sol, hijas del Cid, a manos de sus maridos, los Infantes de Carrión que, vengando así el escarnio sufrido por su cobardía, las abandonan malheridas en el robledal de Corpes. Más adelante, en el siglo XVII, la escritora María de Zayas se sirve de su pluma para retratar en sus Novelas amorosas y ejemplares y en sus Desengaños amorosos a unas mujeres maltratadas física y psicológicamente y sometidas a todo tipo de vejaciones (Sánchez, 2OO3: I49-I84).
} 
la literatura se ha convertido en uno de los campos de exploración para realizar los trabajos de prospección de la relación entre la cultura masculina y femenina y para desenterrar los vestigios de los sistemas de valores que han operado en la marginación y explotación femeninas (Sánchez, 2003: I5I ${ }^{8}$.

El proyecto que presentamos se ha elaborado, siguiendo los principios de la ginocrítica, a partir de la selección de tres obras literarias publicadas en épocas diferentes. Sus autoras son mujeres con unas particulares trayectorias vitales y profesionales de las que, no obstante, solo vamos a ofrecer unas breves pinceladas con el fin de contextualizar sus textos dentro de su propia producción literaria y en un marco temporal concreto. Todo ello nos permitirá, por un lado, analizar las perspectivas desde las que se trata el tema de la violencia de género en estas obras y, por otro, detectar, valorar y reflexionar sobre la incidencia y la posible evolución o transformación de los factores individuales y socioculturales que rodean a las protagonistas de tales producciones. Asimismo, habría que puntualizar que los textos escogidos destacan por su intenso realismo, lo que propicia una reacción por parte del lector que resulta diferente cuando se trata de otras narraciones que, con el mismo fondo argumental, se acercan al tema con mayores dosis de ficción ${ }^{9}$.

Nuestro punto de partida se sitúa a finales del siglo XIX. Aquí, la figura de Emilia Pardo Bazán sobresale por su constante reivindicación de los derechos de las mujeres, cuyas dificultades para acceder a la cultura y su indefensión en las leyes vigentes ante situaciones de violencia la coruñesa no duda en denunciar en numerosos artículos que vieron la luz en publicaciones periódicas como La Ilustración Artística (Ruiz-Ocaña, 2004). Con el mismo talante, tal y como advierte Paredes (1992: 305-3о6), "su faceta más decididamente progresista fue su constante y activa preocupación por el tema de la promoción social y cultural de la mujer"; una situación que ella misma sufrió a lo largo de su vida personal y profesional. Baste referir al respecto que los eruditos de la época rechazaron en tres ocasiones su candidatura a formar parte de la Real Academia Española.

La crítica ha reconocido su carácter polifacético y prolífico, algo evidente si nos aproximamos a su producción cuentística, lo que la convierte en una de las máximas representantes del género. Los cientos de cuentos que escribió a lo largo de su vida los fue dando a conocer en revistas y periódicos, produciéndose así una dispersión que ha dificultado su cuantificación por parte de los investigadores que han acometido el estudio de su obra. Por otra parte, la riqueza temática también constituye un aspecto reseñable de los cuentos de la Pardo Bazán, lo que ha dado lugar a numerosas clasificaciones. Una

\footnotetext{
${ }^{8}$ En este sentido, habría que poner de manifiesto el silenciamiento al que, por cuestiones de género, han quedado relegadas las mujeres escritoras a lo largo de la historia de la literatura. Estas ausencias femeninas en el canon literario, independientemente del género cultivado, han motivado que en los últimos años hayan visto la luz interesantes estudios críticos, muchos de ellos obras colectivas, con el fin de visibilizar, difundir y poner en valor la producción literaria de las autoras más relevantes de distintas épocas. Mencionamos aquí algunos trabajos representativos al respecto, que incluimos siguiendo una ordenación cronológica: Rosal (2006), Sånchez (2008), Rosal (2OII), Sánchez y Gahete (2OI7), Benegas (2OI7), Burguillos (2OI7), Arriaga (2OI7), Romano y Velázquez (2OI8), Sánchez (2019), entre otros.

9 Nos referimos, por ejemplo, a novelas como la titulada Las damas justas, de Rosana Foncea (2OI7).
} 
de las más populares es la que los divide según diversos centros de interés en Cuentos de Galicia, Cuentos religiosos, Cuentos patrióticos y sociales, Cuentos psicológicos, Cuentos populares, legendarios o fantásticos, Cuentos trágicos y dramáticos y Cuentos de objetos y seres pequeños (Paredes, I979). Entre ellos, para nuestro propósito se han seleccionado cinco composiciones (Paredes, r99o) que giran en torno a distintas formas y contextos en los que la violencia de género puede materializarse.

Así pues, el tema de los celos como desencadenante del crimen pasional se manifiesta, aunque con consecuencias muy diferentes, en los relatos titulados "La puñalada" y “Apólogo". Por otra parte, "El encaje roto" nos descubre los rasgos que caracterizan el perfil de un potencial maltratador. Afortunadamente, su pronta identificación por parte de la protagonista momentos antes de contraer matrimonio evitará la consabida tragedia. El desenlace en "El indulto" es otro muy distinto. En esta ocasión, la autora indaga en la psicología de una mujer que vive constantemente aterrorizada tras denunciar a su marido, que la amenaza antes de entrar en prisión para cumplir condena por el homicidio de su suegra. El indulto del preso y la convicción por parte de la protagonista de la consecución de su juramento propician un final que no deja indiferente al lector. Del mismo modo, intensa fuerza emocional encontramos en "Casi artista", donde la protagonista, tras ser abandonada por su marido, logra sobreponerse a su infortunio. Sin embargo, cuando el bienestar parece haberse instalado en su casa, su marido regresa, dispuesto a instalarse también y con la firme intención de destruir y menospreciar todo lo que ella ha ido logrando con tanto esfuerzo y tesón. Poco a poco, y en sentido creciente, la tensión narrativa del relato va aumentando hasta llegar a su punto culminante, ilustrado con una trágica imagen de la que se hace partícipe al lector ${ }^{\text {Io }}$.

La segunda obra seleccionada, Algún amor que no mate (I996), primera novela de la escritora extremeña Dulce Chacón, abre una línea temática donde el sufrimiento de las mujeres que las protagonizan será ya una constante en su producción narrativa (Servén, 2006, 20IO). En este relato, dedicado a "Ellos”, la autora nos presenta en pocas páginas, pero muy condensadas, una historia sobrecogedora y verosímil donde se sumerge de lleno en el tema del maltrato físico y psicológico llevado hasta sus últimas consecuencias.

Una de las singularidades de la novela radica en la existencia de varias voces narrativas que, desde perspectivas diferentes, irán revelando unos hechos estructurados en breves capítulos sin linealidad temporal (Llorente, 2OII). En ellos serán claramente reconocibles las fases del maltrato y su evolución, así como los rasgos prototípicos tanto de la víctima, como de un maltratador cuya personalidad se transformará alternativamente según su escenario de actuación (Lorente, 200I).

Desde la primera página, la narradora en $\mathrm{I}^{\mathrm{a}}$ persona coincide con la protagonista de la historia, llamada paradójicamente Prudencia. Si esta trata de restar importancia a los hechos que relata, justificando la falta de escrúpulos de un marido al que se somete desde el principio cegada por el enamoramiento, la aparición de la $3 \cdot{ }^{\text {a }}$ persona nos

\footnotetext{
Io Para un comentario crítico de estos y otros cuentos véase Paredes (I979), Baquero (I992) y Noya (20I7).
} 
acercará los episodios más dramáticos y violentos alrededor de los que gira la relación entre ambos. Asimismo, a estas voces narrativas se unirá otra que, en $2 .^{\text {a }}$ persona, interpelará a Prudencia, erigiéndose en la voz de su conciencia.

De igual forma, desfilarán ante el lector una serie de innombrados y desdibujados personajes que se mostrarán indiferentes ante los impactantes hechos que acaecen, descargando todo el peso de la acción en una mujer claramente vulnerable que se verá alienada y superada por tales vivencias y que irá "desgranando episodios de su propia vida mientras su desorientación la empuja a preguntarse, acusarse, condolerse y ensayar diferentes identidades y distancias de lo acontecido, para poder sobrevivir en un ejercicio de memoria nunca explicitado como tal” (Servén, 2006: $5^{86}$ ).

Sin embargo, Prudencia no será la única maltratada en la historia. El contenido de unas cartas nos irá descubriendo los detalles sobre la existencia de una amante, un hijo y nuevos episodios de violencia física y psicológica. Las dos mujeres, con una actitud muy diferente ante la vida, afrontarán su realidad con una huida sin retorno con consecuencias determinantes para ambas.

Por último, la tercera novela, del año 2006, es la titulada La segunda mujer ${ }^{\text {I. }}$. En los cinco capítulos en los que se presenta estructurada, la escritora gallega Luisa Castro nos introduce en la tormentosa y asfixiante relación protagonizada por dos personajes marcados por diferencias generacionales y de clase que resultarán insalvables. Si en la obra de Dulce Chacón el maltrato físico se va alternando con el psicológico prácticamente a partes iguales, aquí predomina este último que, según advierte la propia autora, "está en la novela, pero no como una tesis, sino como el resultado de una relación desigual, en la que uno de los miembros está más desprotegido que el otro" (García, 2006: 350).

La protagonista, Julia Varela, una joven de veintiséis años con un futuro prometedor como escritora, se va viendo arrastrada, casi por inercia, al modo de vida de un reputado catedrático perteneciente a la alta burguesía catalana, Gaspar Ferré, treinta años mayor que ella. Una vez que este consigue atraparla con artimañas de gran señor, comienza entre ambos una lucha encarnizada donde los sentimientos encontrados se mezclarán con situaciones incomprensibles para un lector que va siendo consciente de una realidad ante la que la protagonista que, al igual que Prudencia, termina enajenada tanto física como mentalmente, consigue reaccionar a tiempo.

\footnotetext{
II En el año 2007 destaca la publicación de la novela de Gemma Lienas titulada Atrapada en el espejo. En este caso, la autora se centra en los conflictos emocionales y en los mecanismos de dominación psicológica que dan lugar a una forma de maltrato más difícil de detectar y de demostrar. Además, esta obra resulta del mismo modo interesante desde un punto de vista didáctico para ilustrar que la violencia de género, en cualquiera de sus formas, no es privativa de una clase social concreta ni de un nivel cultural determinado, como también ocurre en la novela de Luisa Castro, sino que hay otros factores que determinan su aceptación, o no, por parte de la mujer que la sufre.
} 


\section{Propuesta didáctica}

A continuación, se presentan los apartados de que consta nuestra propuesta. Tras una previa contextualización, se desarrollan los elementos curriculares y metodológicos propios de toda programación. No obstante, habría que tener en cuenta que se proponen una serie de actividades para un número determinado de sesiones que el docente podrá modificar, reducir o ampliar atendiendo al tiempo de que se disponga, las características del alumnado y la materia concreta en la que se lleve a cabo.

\section{I. Contexto}

El proyecto tiene como destinatarios a un grupo de sesenta estudiantes de $3^{\text {.er }}$ curso del Grado en Educación Infantil de la Universidad de Córdoba. La asignatura en la que se pondría en práctica se denomina "Desarrollo de habilidades lingüísticas y sus didácticas". Se trata de una materia anual de 9 créditos ECTS que se concibe desde una doble vertiente. Por un lado, se trabajan aspectos relacionados con el proceso de enseñanza-aprendizaje de las cuatro destrezas lingüísticas en la Educación Infantil; por otro, se plantean actividades prácticas de muy diversa índole orientadas al desarrollo de la competencia comunicativa del alumnado universitario, lo que permite abordar temas transversales como el que nos ocupa, cuya importancia se justifica a partir de los planteamientos teóricos expuestos en la primera parte de este artículo ${ }^{12}$.

\subsection{Objetivos}

Los objetivos que se pretende alcanzar se presentan formulados desde la perspectiva del docente, unos, y desde la perspectiva de los estudiantes, otros. Son los siguientes:

I. Promover el desarrollo emocional, la prevención de la violencia de género y las relaciones de igualdad entre hombres y mujeres en el ámbito universitario.

2. Fomentar la lectura de los textos literarios desde una perspectiva sociocultural.

3. Definir e identificar las distintas formas en que la violencia de género se manifiesta, así como estereotipos sexistas o discriminatorios.

4. Analizar el papel de la mujer en diferentes periodos de la historia a través de los textos literarios y otras manifestaciones artísticas.

5. Desarrollar las habilidades lingüísticas del alumnado universitario.

6. Fomentar la autonomía y el sentido crítico de los estudiantes durante el proceso de enseñanza-aprendizaje.

7. Desarrollar los valores de tolerancia y respecto a través del trabajo cooperativo.

\footnotetext{
I2 No obstante, este contexto concreto constituye un hipotético marco de aplicación de la propuesta didáctica. En este sentido, dado su carácter amplio, abierto y transversal, la planificación es susceptible de trasladarse a cualquier titulación, curso o universidad.
} 


\subsection{Competencias}

Lejos de plantear un proceso de enseñanza-aprendizaje centrado en la figura del docente, abogamos, siguiendo los planteamientos de De Miguel (2009), por un modelo en el que las competencias que el alumnado tendrá que adquirir, y que habrán de ser evaluadas al finalizar el proceso, constituyen el eje de la planificación, determinando el resto de componentes que integran la propuesta. Las competencias que se desarrollarán a lo largo de las sesiones se dividen en básicas, Universidad, específicas y modulares, y han sido extraídas de la memoria del plan de estudios de Graduado/a en Educación Infantil por la Universidad de Córdoba ${ }^{13}$ :

- Competencia Básica $3\left(\mathrm{CB}_{3}\right)$ : Que los estudiantes tengan la capacidad de reunir e interpretar datos [...] para emitir juicios que incluyan una reflexión sobre temas relevantes de índole social, científica o ética.

- Competencia Básica 4 (CB4): Que los estudiantes puedan transmitir información, ideas, problemas y soluciones a un público tanto especializado como no especializado.

- Competencia Universidad 2 (CU2): Conocer y perfeccionar el nivel de usuario en el ámbito de las TIC.

- Competencia Específica I3 (CEi3): Construir una visión actualizada del mundo natural y social.

- Competencia Modular I3 (CM3.4): Analizar e incorporar de forma crítica las cuestiones más relevantes de la sociedad actual que afectan a la educación familiar y escolar: impacto social y educativo de los lenguajes audiovisuales y de las pantallas; cambios en las relaciones de género e intergeneracionales; multiculturalidad e interculturalidad; discriminación e inclusión social y desarrollo sostenible.

- Competencia Modular zo (CM7.3): Valorar la importancia del trabajo en equipo.

- Competencia Modular 44 (CM9.2): Favorecer las capacidades de habla y de escritura.

- Competencia Modular 45 (CM9.3): Conocer y dominar técnicas de expresión oral y escrita.

\subsection{Temporalización}

La propuesta didáctica se abordará a lo largo de seis sesiones de noventa minutos de duración. Puesto que la lectura de una de las tres obras literarias mencionadas constituye un requisito indispensable para la realización de las actividades que se expondrán en el apartado correspondiente, entre la primera sesión y el resto transcurrirán tres semanas. De este modo, los estudiantes tendrán tiempo suficiente para realizar la lectura asignada, cuya adjudicación tendrá lugar en la primera sesión.

I3 Estas competencias responden al contexto en el que se llevaría a cabo la propuesta, por lo que podrán variar en función de la universidad y titulación en la que esta se desarrolle. 


\section{3·5. Metodología}

La metodología de esta propuesta está basada en el fomento de un aprendizaje procesual, autónomo, reflexivo, significativo, participativo y duradero. Todo ello se conseguirá a partir de variadas estrategias en las que docentes y estudiantes aúnen sinergias. Los primeros, pautando las fases del proyecto y guiando a los alumnos durante el proceso. Por su parte, los estudiantes se implicarán activamente en el logro de un aprendizaje que los convertirá en los protagonistas y principales responsables de la construcción de su propio conocimiento, que habrá de tener una proyección social y compartida.

Las sesiones se desarrollarán durante la clase práctica semanal. En ellas el grupoclase se dividirá en dos medianos, por lo que trabajaremos con dos grupos de treinta alumnos en franjas horarias diferentes. A su vez, estos grupos se distribuirán en seis grupos pequeños de cinco estudiantes cada uno.

A lo largo de estas sesiones se irán proponiendo, en número variable, actividades grupales e individuales. Asimismo, como culminación del proyecto se planteará una tarea final ${ }^{14}$. Como se podrá comprobar en el siguiente apartado, su realización dotará a los alumnos de los conocimientos y estrategias necesarios para afrontar el tema que se aborda de manera sólida y fundamentada. No obstante, el carácter proactivo que las caracteriza los invitará a investigar, reflexionar y crear, a la vez que se familiarizan con distintos lenguajes y tipologías textuales. Todo ello, sin olvidar el uso de las TIC, que contribuirán al desarrollo de la competencia digital, imprescindible en la formación de futuros maestros (Cózar y Roblizo, 20I4, Lores; Sánchez y García, 2OI9).

\subsection{Actividades}

I. ${ }^{\text {a }}$ Sesión

Esta sesión se estructura en las dos fases que se exponen a continuación. Su objetivo fundamental es presentar la propuesta, por un lado y, por otro, llevar a cabo una evaluación diagnóstica sobre las concepciones previas de los alumnos respecto a distintos aspectos relacionados con el eje temático del proyecto, en el que comenzarán a indagar.

Fase I. Presentación del proyecto

El docente presentará al alumnado el proyecto, los objetivos que habrá que alcanzar, las competencias que se desarrollarán, la tarea final (tareas 2-5) ${ }^{15}$ y los criterios e instrumentos de evaluación. Se insistirá, especialmente, en las características del portafolio digital que habrá que elaborar a lo largo de todo el proceso ${ }^{16}$. El hecho de que los estudiantes conozcan esta información desde el principio repercutirá positivamente en

\footnotetext{
${ }^{\text {I4 }}$ Distinguimos aquí entre actividades y tareas. Las primeras conllevan la aplicación de procesos mentales por parte del alumnado con el fin de adquirir conocimientos nuevos o utilizar los que ya se poseen en relación con el tema. Su consecución propiciará el logro de los objetivos correspondientes. En cuanto a las tareas, las concebimos desde un nivel superior. En ellas se llevará a cabo un producto final contextualizado para desarrollar las competencias establecidas.

I5 Siguiendo un criterio de claridad expositiva, en este artículo detallaremos las características de la tarea final en la sesion en la que los alumnos tendrán que realizarla.

${ }^{16}$ Sobre ello, véase apartado 3·7. Evaluación.
} 
su motivación, considerada como una estrategia fundamental del aprendizaje.

También en esta fase se configurarán los agrupamientos y se asignarán las lecturas. En este sentido, las tres obras literarias se irán adjudicando por grupos alternativamente, de manera que dos grupos leerán los cuentos seleccionados de Emilia Pardo Bazán, dos la novela de Dulce Chacón y el resto la de Luisa Castro. Se procederá del mismo modo con los seis grupos restantes.

Fase 2. Activación de conocimientos previos

En primer lugar, y de forma individual, los estudiantes tendrán que completar un formulario de Google Drive ${ }^{\mathrm{I} z}$ con cuestiones sobre los estereotipos sexistas, la igualdad entre el hombre y la mujer y su posible repercusión en el uso del lenguaje, los distintos tipos de violencia de género y sus manifestaciones, entre otras.

Seguidamente, distribuidos en grupos de trabajo, los discentes buscarán, seleccionarán y analizarán la forma y el contenido de dos campañas publicitarias sobre la prevención de la violencia de género, una de ámbito nacional y otra internacional, por un lado, y de una obra de arte en la que se manifieste este tema, por otro. Por último, harán lo propio con la letra de una canción. Para ello, podrán recurrir a internet, revistas, periódicos, vallas publicitarias, etc. Esto les permitirá aproximarse a la manera en la que se trata el tema desde distintos ámbitos y al tipo de mensaje, tanto visual como lingüístico, que se transmite. Una vez concluida, añadirán esta actividad al portafolio.

\section{2. ${ }^{\text {a }}$ sesión}

En esta sesión, centrada en una aproximación de carácter teórico al tema, los distintos grupos de trabajo tendrán que elaborar, y añadir al portafolio, el resumen de varios documentos facilitados previamente por el docente en los que se aclara terminológicamente el concepto de la expresión "violencia de género", su tipología y manifestaciones, y se incide en los mecanismos del maltrato psicológico. Son los siguientes: Real Academia Española (2004); Ley I3/2007, de 26 de noviembre, de medidas de prevención y protección integral contra la violencia de género: artículo I bis. "Concepto de víctima de violencia de género" y artículo 3 . "Concepto, tipología y manifestaciones de violencia de género”; Blázquez; Moreno y García-Baamonde (20I5).

\section{$3 \cdot{ }^{\mathrm{a}}$ sesión}

Cada grupo pequeño, en función de la obra literaria que le haya sido asignada, deberá llevar a cabo una investigación (a la que también se dedicará la siguiente sesión), estructurada a partir de los apartados que a continuación se indican:

I. Síntesis del contexto histórico, social y cultural en el que se publican las obras propuestas (siglos XIX, XX y XXI), con especial incidencia en el papel des-

\footnotetext{
I7 Sugerimos como modelo, susceptible de adaptación, el formulario incluido por Romero (2OI7:253) en su propuesta didáctica sobre la concienciación de la violencia de género en el aula de literatura, dirigida a alumnos de ESO, Bachillerato y Enseñanza para Adultos.
} 
empeñado por la mujer en tales ámbitos.

2. Elaboración de una ficha biobibliográfica de la escritora correspondiente (Emilia Pardo Bazán, Dulce Chacón o Luisa Castro).

3. Recopilación de testimonios, orales o escritos, de estas autoras sobre sus propias obras, el papel de la mujer en la sociedad, la violencia de género, etc.

$4 \cdot{ }^{\mathrm{a}}$ sesión

En esta sesión se ampliará el trabajo de investigación iniciado en la $3 \cdot{ }^{\mathrm{a}}$ sesión con las siguientes actividades que, junto con las anteriores, habrá que integrar al portafolio:

4. Resumen del argumento de la obra leída.

5. Selección de 4-5 fragmentos que se consideren especialmente representativos y justificación de su elección.

6. Configuración de una tabla donde se especifique de manera sintética, pero suficientemente ejemplificada, la siguiente información:

o Tipo de violencia que se manifiesta en la obra.

o Rasgos que configuran el perfil de la protagonista.

o Rasgos que configuran el perfil del antagonista.

o Desenlace.

7. Reflexión y concreción de los logros alcanzados y de las posibles dificultades que hayan podido surgir durante el trabajo llevado a cabo en las sesiones anteriores. Además, se determinarán las estrategias utilizadas para solventarlas.

$5 \cdot{ }^{\text {a }}$ sesión

Una vez clarificados y fundamentados algunos aspectos desde el punto de vista teórico, y contextualizadas y analizadas las obras y sus autoras, resulta conveniente ir tejiendo redes intertextuales. Para ello, a partir de los fragmentos seleccionados con anterioridad por cada grupo de trabajo, se llevará a cabo una tertulia literaria dialógica (Flecha, I997) con el grupo mediano. Esta experiencia, basada en el diálogo igualitario y la interacción, permitirá la construcción intersubjetiva del significado de los textos desde una dimensión social de la lectura, lo que redundará en la consiguiente transformación cultural propugnada por el aprendizaje dialógico (Valls; Soler y Flecha, 20o8, Aubert; Flecha; García; Flecha y Racionero, 2008).

Finalizada la tertulia, cuya duración será estimada por el docente en función del grado de participación, los alumnos comenzarán a organizar su trabajo en torno a la tarea final (no presencial y grupal). Se proponen cinco tareas que, excepto la I, común para todos los grupos y a la que se dedicará la siguiente sesión, se explicaron a los estudiantes en la fase i de la I. ${ }^{a}$ sesión. Cada grupo de trabajo tendrá que elegir dos tareas del resto de las opciones $(2,3,4$ y 5$)$ y añadirlas al portafolio del proyecto:

I. Organización de un evento universitario TEDx.

2. Escritura de un texto argumentativo sobre "La prevención de la violencia de género en el ámbito educativo”. Extensión máxima: 8oo palabras. 
3. Elaboración de un tríptico informativo sobre la violencia de género dirigido al alumnado universitario.

4. Realización de una campaña publicitaria sobre la violencia de género. Duración máxima del anuncio: I minuto y 30 segundos.

5. Escritura de un microrrelato sobre el tema del maltrato físico o psicológico. Extensión máxima: I5o palabras. En este sentido, se fomentará la participación del alumnado en el concurso de tuits, microrrelatos y vídeos de un minuto que anualmente se convoca desde la Unidad de Igualdad de la Universidad de Córdoba.

\section{6. ${ }^{\mathrm{a}}$ sesión}

Como se ha adelantado anteriormente, las particularidades de la tarea I han motivado que se destine una sesión monográfica a la misma, donde tendrá lugar tanto su instrucción como su planificación. Esta consiste en la organización de un evento universitario TEDx, que se celebrará en el Salón de Actos de la Facultad de Ciencias de la Educación en el último mes del periodo lectivo de la asignatura.

Básicamente, los objetivos de la plataforma TED (Technology, Entertainment, Design) son inspirar y estimular las mentes a través de la organización de eventos que, con formatos diversos, pretenden generar ideas con impacto en la sociedad. En este caso, el evento se estructurará en doce charlas (una por cada grupo de trabajo) de no más de quince minutos de duración ${ }^{18}$. El tema será el mismo que el de la propuesta didáctica, y las intervenciones deberán versar sobre cualquier aspecto relacionado con la temática en cuestión, pudiendo este no haber sido tratado en las sesiones de manera específica.

Una vez facilitadas estas consignas básicas, serán los estudiantes quienes tengan que gestionar todo lo relacionado con el evento: elaborar el programa, solicitar una licencia TEDx, configurar los equipos y asignar roles, promocionar el acto, etc. En este sentido, será indispensable configurar, y añadir al portafolio, un cuestionario mediante el que los participantes valorarán tanto la organización del evento como la calidad de las charlas. Asimismo, será necesaria la coordinación entre los dos grupos medianos que conforman el grupo-clase, pues el evento, que tendrá lugar en un solo día, será único.

\subsection{Evaluación}

Se plantea una evaluación formadora con el fin de favorecer, mediante una serie de estrategias e instrumentos, que el estudiante sea consciente de su aprendizaje no solo al final, sino en todo momento, al tiempo que desarrolla su habilidad para aprender a aprender (Bordas y Cabrera, 20OI) ${ }^{19}$. En este sentido, nos adherimos a la concepción actual de la evaluación (Bordas y Cabrera, 2OOI, Sanmartí, 20O7, Álvarez, 2008, Álvarez,

\footnotetext{
${ }^{18}$ Sobre ello, se aconseja la lectura de la guía oficial de TED para hablar en público (Anderson, 20I6).

I9 Las autoras abordan las diferencias entre la evaluación formativa y la formadora. Si la primera depende de la intervención e iniciativa del docente y tiene un carácter extrínseco, al provenir de fuera, la evaluación formadora, orientada por el docente, parte de la iniciativa del propio discente, por lo que se identifica por su carácter intrínseco; aspecto que garantiza el aprendizaje.
} 
2OII, 2OI2, Brown y Pickford, 2OI3, Arribas, 2OI7) desde la que se propugna su integración en el proceso de enseñanza-aprendizaje, lo que conlleva "una mayor comprensión tanto por parte del profesor como del estudiante de los procesos que se están realizando, así como el conocimiento de las razones de los errores y aciertos que se producen" (Bordas y Cabrera, 2OOI: 32).

En cuanto a los agentes de la evaluación, estos serán tanto el profesor como los alumnos. Así, más allá del carácter bidireccional que supone la tradicional evaluación profesor-alumno (heteroevaluación), los estudiantes se verán implicados activamente, convirtiéndose en los responsables de la autorregulación de su aprendizaje y del de sus compañeros. Atendiendo a esta premisa, se contempla la autoevaluación y la evaluación entre iguales (coevaluación) como procesos para la valoración de la competencia en el trabajo en equipo ${ }^{20}$, pero también para determinar el desarrollo del resto de competencias de la propuesta didáctica.

Para ello, consideramos que el instrumento que mejor se adapta a este enfoque es el portafolio digital, cuyo valor evaluativo y pedagógico, según Barragán (2005: I23), "permite valorar no solo lo aprendido, sino también la capacidad de aprendizaje que se demuestra y las habilidades que se tienen en ello". Por su parte, Barberà; Bautista; Espasa y Guasch (2006: 56) lo definen como "una selección de evidencias/muestras (que forman un dossier o una carpeta) que tiene que recoger y aportar el estudiante a lo largo de un período de tiempo determinado y que responde a un objetivo concreto". Además, los mismos autores aluden a la necesidad de que tales evidencias se acompañen "de una justificación y una reflexión del estudiante, en que ponga de manifiesto la relación entre la evidencia y el aprendizaje" (Barberà et al., 2006: 56). Precisamente, serán los alumnos quienes se responsabilicen de su diseño y organización, para lo que deberán utilizar el formato de presentación que consideren más adecuado.

El hecho de que se trate de un portafolio digital facilita no solo su estructuración, que deja de ser lineal en relación con el portafolio tradicional, sino también el tipo de materiales que se pueden integrar (textos, imágenes, vídeos, elementos multimedia, etc.). Además, su carácter abierto y accesible, tanto para los alumnos como para el profesor, favorecerá que los primeros, de manera autónoma, crítica y reflexiva, introduzcan las mejoras oportunas y vayan dando respuesta a las indicaciones del docente que, de forma periódica, revisará las actividades y tareas que lo integran, aportando la retroalimentación necesaria de cara a la versión final ${ }^{22}$.

Por último, para la valoración de la competencia comunicativa, el alumnado tendrá a su disposición en el portafolio digital una e-rúbrica específica, lo que le permitirá enfrentarse a las actividades y tareas correspondientes de una forma mucho más consciente, al conocer desde el principio qué y cómo se valorará su nivel de desempeño (Cano, 20I5).

\footnotetext{
${ }^{20}$ Como sugerencia, los alumnos podrían cumplimentar, y añadir al portafolio, los cuestionarios destinados a tal fin propuestos por Jiménez-Tenorio; Aragón; Sánchez; Aragón; Azcárate; Cardeñoso y Moreno (2OI4: II-I2).

${ }^{2 \mathrm{I}}$ García (2005: II5) aclara que el aspecto que diferencia un portafolio digital de otro que no lo es radica en el "uso interactivo del mismo. Por lo tanto, un portafolio electrónico debe tener un marcado componente de interactividad que permita usos y aplicaciones muy difíciles o prácticamente imposibles en un portafolio de papel y lápiz". Sobre su estructura, véase Barberà; Bautista; Espasa y Guash (2006).
} 


\section{Conclusiones}

Este trabajo surge a partir de la necesidad de desarrollar en las aulas universitarias la competencia transversal sobre la violencia de género y la igualdad entre hombres y mujeres, tal y como se demanda desde la legislación vigente. Ante la constatada escasez de propuestas didácticas en el ámbito de actuación que nos compete, se ha pretendido, por tanto, ofrecer una herramienta que permita a los docentes formar, sensibilizar y educar, en el más amplio sentido del término, al alumnado universitario, futuros educadores, en nuestro caso, que han de tener una clara y sólida conciencia de la importancia de transformar la realidad.

La fundamentación teórica que se incluye en la primera parte presenta un actualizado estado de la cuestión desde un punto de vista legislativo, pero también de las principales investigaciones que, desde diversos ámbitos, se han llevado a cabo en relación con el tema que nos ocupa y que ponemos en relación con la literatura como producto histórico, cultural y social en la segunda parte. En ella hemos presentado un proyecto que, como se habrá podido constatar, pretende ir más allá del mero cumplimiento de una ley. En este sentido, cabe puntualizar que, con su puesta en práctica, no se persigue la enseñanza de la Literatura Española como tal, pues, de ser así, su orientación y las actividades que lo conforman habrían sido otras. Por el contrario, la obra literaria constituye un valioso recurso para guiar a sus receptores en el proceso de construcción, de manera pautada, crítica, fundamentada y compartida, de su propio conocimiento acerca del tema.

Para el logro de los objetivos propuestos se han seleccionado textos que, partiendo del siglo XIX hasta la época actual, reflejan, desde la óptica de sus autoras, el tradicional modelo patriarcal, además de distintos contextos en los que el maltrato se establece. De este modo, las obras elegidas presentan las historias de personajes con unos perfiles muy diferentes que reaccionan de diversas formas ante sus particulares circunstancias, lo que favorece la indagación, a su vez, en variados mecanismos de violencia cuyas repercusiones resultan evidentes para los lectores, aportándoles así una visión integral sobre ello.

En cuanto a las actividades, la manera en la que se presentan secuenciadas en las distintas sesiones contribuirá a la adquisición gradual de los aprendizajes. De esta forma, las correspondientes a la primera sesión aportarán al alumnado los conocimientos teóricos necesarios para la construcción de una opinión sólidamente fundamentada en torno al tema. Si las incluidas en la segunda sesión propiciarán una aproximación al contexto histórico, social y cultural de las obras literarias, así como a la trayectoria de sus autoras, en la tercera se profundiza en el contenido de los textos. Ya en la cuarta, retomando lo aprendido, el establecimiento de redes intertextuales requerirá la activación de los conocimientos de diversa índole adquiridos previamente que, a su vez, habrán de materializarse en la tarea final.

Además, estas actividades y tareas también favorecerán el alcance de otros objetivos y el desarrollo de competencias específicas en el marco de la asignatura en la que el 
proyecto se llevaría a cabo, si bien es cierto que su carácter flexible y abierto posibilitaría su planteamiento en cualquier otra materia, con las adaptaciones que el docente responsable considere oportunas. De esta manera, se ha procurado alternar los agrupamientos, desarrollar las distintas destrezas lingüísticas, fomentar la creatividad, la motivación, el uso de herramientas y aplicaciones tecnológicas, que los alumnos se familiaricen con distintas tipologías textuales y, por tanto, con sus respectivos lenguajes, etc.

En definitiva, se trata de contribuir, desde las buenas prácticas en la docencia universitaria, a la formación de futuros profesionales, pero también, y sobre todo, de ciudadanos responsables, comprometidos y conscientes de la importancia de terminar con una lacra social que, como pone de manifiesto el mundo literario configurado en torno al tema, así como el mundo real, lejos de disminuir, parece acrecentarse con el paso del tiempo. 


\section{Referencias bibliográficas}

- Aguilar, C.; Alonso, M. J.; Melgar, P. y Molina, S. (2009). Violencia de género en el ámbito universitario. Medidas para su superación. SIPS. Revista interuniversitaria de Pedagogía Social, I6, 85-94.

- Álvarez, J. M. (2OII). Evaluar para conocer, examinar para excluir. Madrid: Morata.

- Álvarez, J. M. (2012). Didáctica, currículo y evaluación: ensayos sobre cuestiones didácticas. Buenos Aires: Miño y Dávila.

- Álvarez, I. (2008). Evaluación del aprendizaje en la Universidad: una mirada retrospectiva y prospectiva desde la divulgación científica. Revista Electrónica de Investigación Psicoeducativa, I4, 235-272.

-Anderson, C. (20I6). TED Talks. The Official TED Guide to Public Speaking. London: Headline.

-Arriaga, M. (Ed.) (20I7). Escritoras en torno al canon. Sevilla: Benilde.

-Arribas, J. M. (20I7). La evaluación de los aprendizajes. Problemas y soluciones. Profesorado. Revista de currículum y formación del profesorado, 4, 381-404.

- Aubert, A.; Flecha, A.; García, C.; Flecha, R. y Racionero, S. (2008). Aprendizaje dialógico en la sociedad de la información. Barcelona: Hipatia Editorial.

- Bajtín, M. (I99I). Teoría y estética de la novela. Madrid: Taurus.

- Baquero, M. (I992). El cuento español en el siglo XIX. Madrid: CSIC.

- Barberà, E.; Bautista, G.; Espasa, A. y Guasch, T. (2006). Portfolio electrónico: desarrollo de competencias profesionales en la red. RUSC. Revista de Universidad y Sociedad del Conocimiento, $2,55^{-66 .}$

- Barragán, R. (2005). El portafolio, metodología de evaluación y aprendizaje de cara al nuevo Espacio Europeo de Educación Superior. Una experiencia práctica en la Universidad de Sevilla. Revista Latinoamericana de Tecnología Educativa, 4, I2I-I39.

- Benegas, N. (20I7). Ellas tienen la palabra. Las mujeres y la escritura. Madrid: Fondo de Cultura Económica de España.

- Blázquez, M.; Moreno, J. M. y García-Baamonde, M. E. (20I5). Maltrato psicológico en las relaciones de pareja. La inteligencia emocional como factor protector y diferencias de género. Boletín de Psicología, п氵3, 29-47. 
- Bordas, M. I. y Cabrera, Flor Á. (2OOI). Estrategias de evaluación de los aprendizajes centrados en el proceso. Revista Española de Pedagogía, 218, 25-48.

- Brown, S. y Pickford, R. (20I3). Evaluación de habilidades y competencias en Educación Superior. Madrid: Narcea.

- Burgués, A.; Oliver, E.; Redondo, G. y Serrano, A. (2006). Investigaciones mundiales sobre violencia de género en la universidad. En M. García; A. Calvo; J. M. Osoro y S. Rojas (Coords.). Convergencia con Europa y cambio en la universidad: XI Conferencia de Sociología de la Educación, Santander, 22-24 de septiembre de 2005 (I3O-I3I). Alzira (Valencia): Germania.

- Burguillos, M. (Ed.) (20I7). Escritoras, silencios y contracanon. Sevilla: Benilde.

- Calero, M. A.; Molina, M.; Bardina, Y.; Murillo, M. y Rodríguez, C. (2013). Percepción de la violencia de género en el entorno universitario. El caso del alumnado de la Universidad de Lleida. Lleida: Universidad de Lleida.

- Cano, E. (20I5). Las rúbricas como instrumento de evaluación de competencias en Educación Superior: ¿uso o abuso? Profesorado. Revista de currículum y formación de profesorado, $2,265^{-280}$.

- Castro, L. (2006). La segunda mujer. Barcelona: Seix Barral.

- Chacón, D. (1996). Algún amor que no mate. Barcelona: Planeta.

- Cózar, R. y Roblizo, M. J. (2OI4). La competencia digital en la formación de los futuros maestros: percepciones de los alumnos de los grados de maestro de la Facultad de Educación de Albacete. RELATEC. Revista Latinoamericana de Tecnología Educativa, 2, II9-I33.

- De Miguel, M. (Coord.) (2009). Metodologías de enseñanza y aprendizaje para el desarrollo de competencias. Orientaciones para el profesorado universitario ante el Espacio Europeo de Educación Superior. Madrid: Alianza Editorial.

- Díez, J. L.; Cerezo, A. I. y Benítez, M. J. (20I8). La política criminal contra la violencia sobre la mujer pareja (2004-2014). Su efectividad, eficacia y eficiencia. Valencia: Tirant lo Blanch.

- Fernández, R. (20I4). Igualdad, violencia de género y salud en la Universidad de Málaga. Tesis doctoral. Málaga: Universidad de Málaga.

- Flecha, R. (1997). Compartiendo palabras. El aprendizaje de las personas adultas a través del diálogo. Barcelona: Paidós. 
- García, F. (2005). El papel de los portafolios electrónicos en la enseñanza-aprendizaje de lenguas. Glosas didácticas. Revista Electrónica Internacional de Didáctica de las Lenguas y sus Culturas, I4, II2-II9. Obtenido el or de marzo de 2019 desde https://www.um.es/ glosasdidacticas/GDi4/ıo.pdf

- García, V.; Fernández, A.; Rodríguez, F. J.; López, M. P. y Lana, A. (2013). Violencia de género en estudiantes de enfermería durante sus relaciones de noviazgo. Atención Primaria, $6,290-296$.

- García, L. (2006). Luisa Castro, "La segunda mujer”. Barcarola. Revista de creación literaria, 68-69, 347-35'.

- Giménez, P. (2007). Un estudio de la IV Conferencia Mundial sobre las mujeres. Revista de Comunicación y Hombre, 3, 8I-94.

- Goldmann L. (1975). Para una sociología de la novela. Madrid: Ayuso.

- González, F. y Mora, B. (2OI4). Características de la violencia de género en la Universidad de Valencia. Escritos de psicología, 7, 36-43.

- Hernando, A.; García, A. D. y Montilla, M. V. (2OI2). Exploración de las actitudes y conductas de jóvenes universitarios ante la violencia en las relaciones de pareja. Revista Complutense de Educación, 23, 427-44I.

- Jiménez-Tenorio, N.; Aragón, L.; Sánchez, S.; Aragón, C.; Azcárate, P.; Cardeñoso, J. M. y Moreno, F. (2OI4). La coevaluación-autoevaluación como instrumentos para valorar la competencia en el trabajo de equipo. En IV Jornadas de Innovación Docente. Abriendo caminos para la mejora educativa. Sevilla: Universidad de Sevilla.

- Larena, R. y Molina, S. (2OIo). Violencia de género en las universidades: investigaciones y medidas para prevenirla. Trabajo Social Global, 2, 202-219.

- Ley Orgánica I/2004, de 28 de diciembre, de Medidas de Protección Integral contra la Violencia de Género. Boletín Oficial del Estado. Madrid, 29 de diciembre de 2004, 313, 4216642197. Obtenido el oi de marzo de 2Or9 desde https://www.boe.es/boe/dias/2OO4/I2/29/ pdfs/A42I66-42I97.pdf

- Ley 13/2007, de 26 de noviembre, de medidas de prevención y protección integral contra la violencia de género. Boletín Oficial del Estado. Madrid, i3 de febrero de 2008, 38, 7773-7785. Obtenido el or de marzo de 2019 desde https://www.boe.es/eli/es-an/1/2007/II/26/I3/ $\mathrm{dof} / \mathrm{spa} / \mathrm{pdf}$

-Llorent, M. y Sianes, A. (20I6). Micromachismos coercitivos desde la perspectiva del alumnado universitario: una aproximación descriptiva. En C. García; C. Flecha; M. J. Cala; 
M. Núñez y A. Guil (Coords.). Mujeres e investigación. Aportaciones interdisciplinares: VI Congreso Universitario Internacional "Investigación y Género", Sevilla, 3 o de junio y i de julio de 2016 (385-395). Sevilla: AEMUS.

- Llorente, L. I. (2OII). Voces narrativas en Algún amor que no mate. Espéculo. Revista de Estudios Literarios, 47. Obtenido el oi de marzo de 2019 desde http://www.ucm.es/info/especulo/numero47/algunam.html

- Lorente, M. (200I). Mi marido me pega lo normal. Agresión a la mujer: realidades y mitos. Barcelona: Ares y Mares.

- Lores, B.; Sánchez, P. y García, M. R. (2Or9). La formación de la competencia digital en los docentes. Profesorado. Revista de currículum y formación del profesorado, 4, 234-26o.

- Luckás, G. (ı973). Sociología de la Literatura. Barcelona: Península.

- Moreno, M. A. (20io). La literatura universal como recurso de aula para la prevención de la violencia contra las mujeres. Tabanque, 23, 83-98.

- Muñoz, M.; Graña, J. L.; O 'Leary, K. D. y González, P. (2007). Agresión física y psicológica en las relaciones de noviazgo en universitarios españoles. Psicothema, I9, IO2-IO7.

- Naciones Unidas (1995). Declaración y Plataforma de Acción de Beijing. Obtenido el or de marzo de 2019 desde https://www.acnur.org/fileadmin/Documentos/Publicaciones/2OI5/9853.pdf?file=fileadmin/Documentos/Publicaciones/20I5/9853

- Noya, R. (20I7). La violencia en los cuentos de Emilia Pardo Bazán. Tesis doctoral. Santiago de Compostela: Universidad de Santiago de Compostela.

- Oliver, E. y Valls R. (2004). Violencia de género. Investigaciones sobre quiénes, por qué y cómo superarla. Barcelona: Hipatia Editorial.

- Paredes, J. (i979). Los cuentos de Emilia Pardo Bazán. Granada: Universidad de Granada.

- Paredes, J. (Ed.) (1990). Emilia Pardo Bazán. Cuentos completos. 4 tomos. La Coruña: Fundación "Pedro Barrié de la Maza Conde de Fenosa”.

- Paredes, J. (r992). El feminismo de Emilia Pardo Bazán. Cuadernos de Estudios Gallegos, IO5, 303-3І3.

- Puigvert, L. et al. (2007-20IO). Incidencia de la ley integral contra la violencia de género en la formación inicial del profesorado. Plan Nacional I+D+I. Ministerio de Sanidad, Servicios Sociales e Igualdad. Instituto de la Mujer. Obtenido el or de marzo de 2org desde http:// www.inmujer.gob.es/fr/areasTematicas/estudios/estudioslinea2OI2/docs/Incidencia_ 
Ley_integral.pdf

- Real Academia Española (2004). Informe académico sobre la expresión violencia de género. Panace@, I6, IOо-IOI. Obtenido el or de marzo de 2019 desde http://www.medtrad. org/panacea/IndiceGeneral/nı6_tradyterm_RAE.pdf

- Romano, Y. y Velázquez, S. (Coords.) (20I8). Las inéditas: voces femeninas más allá del silencio. Salamanca: Ediciones Universidad de Salamanca.

- Romero, A. (2OI7). La violencia de género en el aula de literatura. Un proyecto por la sensibilización con la violencia de género en el aula de literatura a través de cuentos de Emilia Pardo Bazán. Didáctica. Lengua y Literatura, 29, 235-258.

- Rosal, M. (2006). Con voz propia. Estudio y antología comentada de poesía escrita por mujeres (1970-2005). Sevilla: Renacimiento.

- Rosal, M. (2OII). Poesía española escrita por mujeres a finales del siglo XX. Itinerarios. Revista de estudios lingüisticos, literarios, históricos y antropológicos, I3, I57-I67.

- Ruiz-Ocaña, E. (2004). Emilia Pardo Bazán y los asesinatos de mujeres. Didáctica (Lengua y Literatura), I6, I77-I88.

- Sánchez, B. (2003). Sobre violencia, opresión y vejaciones en las novelas de María de Zayas. En M. D. Adam y M. J. Porro (Eds.). Violencia y género: congreso internacional g-ıo de marzo de 2001 (I49-I84). Córdoba: Servicio de Publicaciones Universidad de Córdoba.

- Sánchez, B. (2008). De la invisibilidad a la creación. Oralidad, concepción teórica y material preceptivo en la producción literaria femenina hasta el siglo XVIII. Sevilla: Renacimiento.

- Sánchez, R. y Gahete, M. (Coords.) (20I7). La palabra silenciada: voces de mujer en la poesía española contemporánea (I95O-20I5). Valencia: Tirant Lo Blanch.

- Sánchez, R. (20i9). Canon escolar poético y pedagogía literaria en bachillerato. Las escritoras invisibles en los manuales de literatura. Pedagogía Social. Revista Interuniversitaria, $33,43-5^{2}$.

- Sanmartí, N. (2007). 1 ideas clave. Evaluar para aprender. Barcelona: Graó.

- Santos, T.; Bas, E. e Iranzo, P. (2OI2). La formación inicial del profesorado en prevención y detección de la violencia de género: universidades españolas y universidades de prestigio internacional. Revista Interuniversitaria de Formación de Profesorado, 73, 25-39.

- Servén, C. (2006). La narrativa de Dulce Chacón: memoria de las perdedoras. Arbor Ciencia Pensamiento y Cultura, 72I, 583-59I. 
- Servén, C. (2OIO). Literatura y educación en valores: relatos de intriga y violencia de género. Revista Ibero-Americana de Estudos em Educaçao, I, IO-2O.

- Tapia, S. I. (20I5). Violencia de género en las universidades o la necesidad de una intervención educativa. INFAD. Revista de Psicología, I, 53-544.

-Valls, R. et al. (2004-2007). Violencia de género en las universidades españolas. Ministerio de Trabajo y Asuntos Sociales. Instituto de la Mujer. Plan Nacional de I+D+I 2004-2007. Obtenido el or de marzo de 2019 desde http://www.uca.es/recursos/doc/unidad_igual$\mathrm{dad} / 496 \mathrm{IO} 6686 \_4720 \mathrm{III} 25339 \cdot \mathrm{pdf}$

- Valls, R.; Oliver, E.; Sánchez, M.; Ruiz, L. y Melgar, P. (2007). ¿Violencia de género también en las universidades? Investigaciones al respecto. Revista de Investigación Educativa, 25, 2I9-23I.

- Valls, R.; Soler, M. y Flecha R. (2008). Lectura dialógica: interacciones que mejoran y aceleran la lectura. Revista Iberoamericana de Educación, 46, 7 7-87.

-Vázquez, E.; Torres, A.; Otero, P.; Blanco, V. y López, M. (20Iо). Prevalencia y factores de riesgo de la violencia contra la mujer en estudiantes universitarias españolas. Psicothema, 22, I96-2OI. 